\title{
Resolution of Anterior Scleritis after Periodontal Therapy
}

\author{
Guliz Nigar Guncua
}

Feriha Caglayan ${ }^{a}$

\begin{abstract}
Scleritis is a chronic inflammation that involves the outermost coat and the skeleton of the eye. Scleritis may be associated with a systemic or immune mediated disease and it might be caused by an infection, trauma or drug reaction. This case presents a patient with generalized chronic periodontitis and anterior diffuse scleritis. A 30-year-old female complained of pain and persistent scleritis in the left eye was referred to the Periodontology department due to her periodontal problems. She was treated with oral non steroidal anti-inflammatory drugs and topical medications (corticosteroid) for her scleritis during last two years. However, these treatments failed to control the progression of the disease. After periodontal examination, deep periodontal pockets and serious bone loss was detected radiographically. She was treated by flap procedures and 3 teeth were extracted. After a 4-month healing period, scleritis was resulted in rapid resolution. (Eur J Dent 2011;5:337-339)
\end{abstract}

Key words: Anterior scleritis; Periodontitis; Treatment.

\section{INTRODUCTION}

Scleritis is a chronic inflammation that involves the outermost coat and skeleton of the eye and it has a characteristic clinical picture. The scleral vessels are not prominent in a healthy eye; however, in scleritis the vascular structure is marked. This form of ocular inflammation can lead to vision-threatening complications, including uveitis,

a Department of Periodontology, Faculty of Dentistry, Hacettepe University, Ankara, Turkey.

- Corresponding author: Dr. Guliz N. Guncu Department of Periodontology, Faculty of Dentistry Hacettepe University, Sihhiye, TR-06100, Ankara, Turkey. Phone: +903123052237

Fax: +90 3123104440

E-mail: gulizahacettepe.edu.tr cataract, and glaucoma, as well as various retinal pathologies that can be extremely painful., ${ }^{1,2}$ Scleritis may involve either the anterior sclera or posterior sclera or both. Anterior scleritis is the most common form and it can be diffuse (the entire circumference of the sclera is involved), nodular (presented as an inflamed nodule on the surface of the sclera), or necrotizing (complicated by necrosis of the sclera, which can be associated with active inflammation or can occur otherwise in non-inflamed sclera). 1,3,4

Scleritis can occur as a condition confined to the eye or it might be associated with a systemic, immune-mediated disease; uncommonly, scleritis might be caused by an infection or drug reaction, 
or by an occult (hidden) tumor, or complications following surgery. ${ }^{4}$ Inflammatory bowel disease is a chronic inflammatory disorder that is characterized by chronic inflammation of the intestine. ${ }^{5}$ Inflammatory bowel disease patients might have scleritis in up to $18 \%$ of cases and also $2 \%$ of scleritis patients have inflammatory bowel disease. ${ }^{5,6}$ Therefore, one may suggest a close relationship between systemic infection/inflammation and scleritis.

This case presents a patient with periodontitis and anterior diffuse scleritis and points out the possible relationship between scleritis and periodontal infection, and inflammation.

\section{CASE REPORT}

A 30-year-old female complained of pain and persistent scleritis in the left eye and was referred to the Periodontology department due to her periodontal problems (Figure 1). She had scleritis for 13 months. At that time, she was evaluated with her complete medical history and review of symptoms; particularly the symptoms of infectious and rheumatic diseases were targeted. According to medical evaluation, complete blood count parameters were within normal limits, except slight leukocytosis [Leukocyte $11.3 \times 10^{3} / \mu \mathrm{L}$ (normal range: $\left.\left.4.5-11 \times 10^{3} / \mu \mathrm{L}\right)\right]$. Additionally, all other parameters were in normal levels and erythrocyte sedimentation rate, C-reactive protein (CRP), complement C3, complement C4, rheumatoid factor, anti-ds DNA, antinuclear antibody (ANA) profile, antineutrophil cytoplasm antibody (ANCA) profile, and anti-gliadin antibodies were detected as negative. Rheumatologic evaluations were normal.

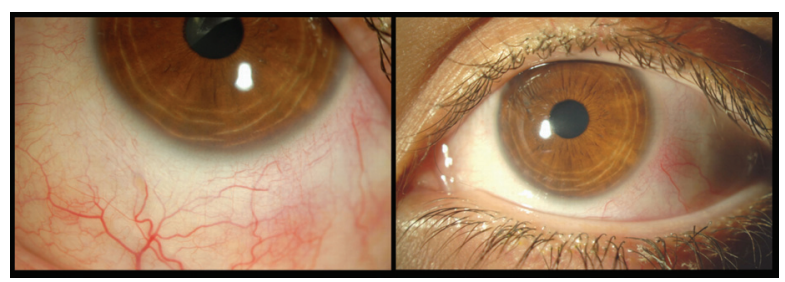

Figure 1. Clinical appearance of anterior diffuse scleritis in the left eye.

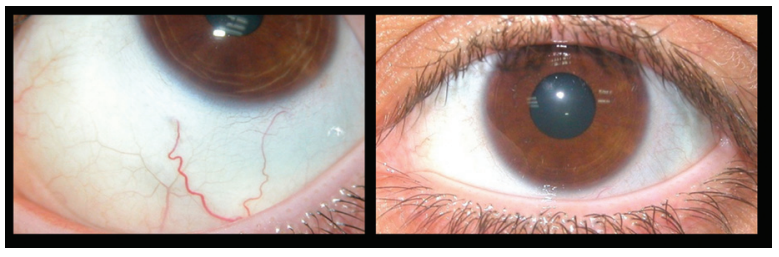

Figure 3. Clinical view of resolution of scleritis 4 months after periodontal therapy.
Her doctor advised to use oral non steroidal antiinflammatory drugs and later ophthalmologic steroid solutions. However, these treatments failed to control the progression of the disease, and the symptoms of scleritis sometimes decreased then increased again. A year after the initial diagnosis, she was referred to the Periodontology department. After periodontal examination, deep periodontal pockets and serious bone loss was detected radiographically (Figure 2). The diagnosis was generalized as chronic periodontitis. Flap procedure was performed in 3 quadrants (right and left maxillary quadrants and left mandibular quadrant), and the maxillary right second premolar, maxillary left third molar, and mandibular left second premolar teeth were extracted due to the serious bone loss. Additionally, the mandibular left third molar was also extracted. After a healing period, scleritis was resulted in rapid resolution (Figure 3). The patient was re-evaluated after 6 months and no recurrence in scleritis or periodontitis was detected.

\section{DISCUSSION}

It is well documented that many systemic conditions may affect the oral cavity. In contrast, current theories speculate that the oral conditions may negatively affect systemic health. ${ }^{7}$ The subgingival microbiota in patients with periodontitis provides a significant and persistent gramnegative bacterial challenge to the host. It has been noted that periodontal pathogens are able to invade gingival tissues, ${ }^{8}$ and from there gain access to the systemic circulation. ${ }^{9}$ Additionally, immunoinflammatory response to bacteria and

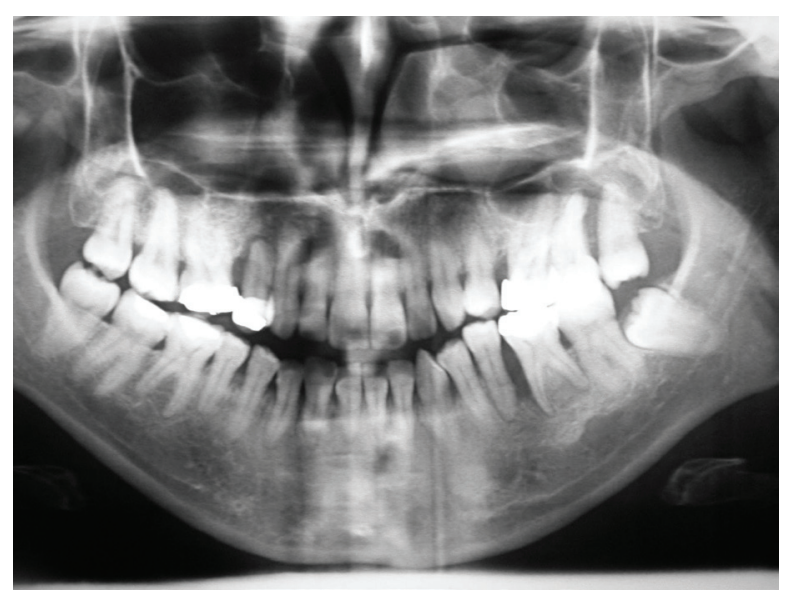

Figure 2. Serious bone loss in the maxillary left and right and mandibular right premolar and molar regions. 
their byproducts occurs in the periodontal tissues; systemic challenge with these agents also induces a major vascular response. Furthermore, subjects with periodontitis present changes in their systemic inflammatory parameters: not only do periodontitis patients have increased local periodontal inflammation but they also have higher inflammation in their bloodstream. When compared with periodontally healthy subjects, patients with periodontitis have higher numbers of blood granulocytes or higher concentrations of acute-phase markers such as CRP, fibrinogen, and a host of cytokines. ${ }^{9}$

This case report presents the resolution of ophthalmologic complication following the treatment of periodontal inflammation. It may be suggested that increased systemic inflammation related to periodontitis may have played a role as the initiating factor for scleritis. Ophthalmic complications like scleritis are usually of inflammatory origin and may reflect the overall disease activity. ${ }^{10}$ Moreover, in most patients the underlying disease precedes the scleritis and the physicians should suspect this from the pertinent nonocular symptoms such as rheumatoid arthritis, Wegener's granulomatosis, systemic lupus erythematosus, and inflammatory bowel disease. ${ }^{1}$

Similar to inflammatory bowel disease, periodontitis is also a chronic inflammatory disease. In a recent meta-analysis, it was found that subjects with periodontitis have higher serum CRP concentrations as compared with subjects without periodontitis. ${ }^{11}$ Furthermore, it was shown that periodontal therapy consisting of oral hygiene instructions, scaling and root planing, and extraction of hopeless teeth can result in a reduction in the inflammatory markers (interleukin-6 and CRP). ${ }^{12}$ In the present case, it may be concluded that treatment of periodontal disease led to a decrease in the inflammatory markers and scleritis resolution. Therefore, according to literature and from our case study, it may be speculated that anterior scleritis may be initiated by increased systemic inflammation caused by periodontal disease.

\section{CONCLUSIONS}

When treatment of scleritis with non steroidal anti-inflammatory drugs or corticosteroid-sparing agents failed, and the patient has no systemic or immune-mediated disease such as rheumatoid arthritis or Wegener's granulomatosis, the dentist should consider a probable periodontal inflammation as an etiology of scleritis.

\section{REFERENCES}

1. Smith JR, Mackensen F, Rosenbaum JT. Therapy insight: scleritis and its relationship to systemic autoimmune disease. Nat Clin Pract Rheumatol 2007;3:219-226.

2. Watson PG, Hayreh SS, Awdry PN. Episcleritis and scleritis. I. Br J Ophthalmol 1968;52:278-279.

3. Watson PG, Hayreh SS. Scleritis and episcleritis. Br J Ophthalmol 1976;60:163-191.

4. Pavesio CE, Meier FM. Systemic disorders associated with episcleritis and scleritis. Curr Opin Ophthalmol 2001;12:471478.

5. Ghanchi FD, Rembacken BJ. Inflammatory bowel disease and the eye. Surv Ophthalmol 2003;48:663-676.

6. Knox DL, Schachat AP, Mustonen E. Primary, secondary and coincidental ocular complications of Crohn's disease. Ophthalmology 1984;91:163-173.

7. Garcia RI, Henshaw MM, Krall EA. Relationship between periodontal disease and systemic health. Periodontol 2000 $2001 ; 25: 21-36$.

8. Papapanou PN, Sandros J, Lindberg K, Duncan MJ, Niederman R, Nannmark U. Porphyromonas gingivalis may multiply and advance within stratified human junctional epithelium in vitro. J Periodontal Res 1994;29:374-375.

9. Tonetti MS. Periodontitis and risk for atherosclerosis: an update on intervention trials. J Clin Periodontol 2009;36 Suppl 10:15-19.

10. Okhravi N, Odufuwa B, McCluskey P, Lightman S. Scleritis. Surv Ophthalmol 2005;50:351-363.

11. Paraskevas S, Huizinga JD, Loos BG. A systematic review and meta-analyses on $\mathrm{C}$-reactive protein in relation to periodontitis. J Clin Periodontol 2008;35:277-290.

12. D'Aiuto F, Parkar M, Andreou G, Suvan J, Brett PM, Ready $D$, et al. Periodontitis and systemic inflammation: control of the local infection is associated with a reduction in serum inflammatory markers. J Dent Res 2004;83:156-160. 